\title{
Stabilization of transport tracked vehicle trajectory
}

\author{
Igor Taratorkin ${ }^{1,2, *}$, Victor Derzhanskii ${ }^{1,2}$, and Alexander Volkov ${ }^{1}$ \\ ${ }^{1}$ IES UB RAS, Department of Transportation Vehicles Mechanics, 620049, Ekaterinburg, Russia \\ ${ }^{2} \mathrm{KSU}$, Kurgan State University, Department of Applied Mechanics , 640020, Kurgan, Russia
}

\begin{abstract}
The article presents the research findings of the controlled motion dynamics of tracked vehicles equipped with a steering system with discrete properties. It is established that the potential high-speed performance is limited by motion instability and by dynamic properties i.e. the phase lag of the vehicle response to the harmonic control input and the "engine overshoot" to a unit step function (steering jerk). Technical proposals allowing for the high-speed performance of the vehicle are substantiated, such as yaw moment control, which ensures the positivedifference of the partial differential coefficients of yaw moment and cornering resistance moment with respect to curvature; increase of the dynamic system stiffness for increasing the natural frequency and decreasing energy when exciting oscillatory processes; implementation of Shaper steering brake control algorithms.
\end{abstract}

\section{Introduction}

One of the main operational properties of transport tracked vehicles is high-speed performance, which is evaluated by speed characteristics. Increase in power-to-weight ratio of vehicles up to $25 \mathrm{~kW} / \mathrm{t}$, improvement of transmissions, motion control systems and information applications will increase the potential high-speed properties of modern tracked vehicles. However, their implementation is limited by a number of dynamic phenomena that characterize controllability at rectilinear motion and at turning, especially when applying vehicle robotization (with remote or application control). Most modern vehicles are equipped with the steering control system with continuous properties provided by hydrostatic transmissions (HST). Their main drawback is that to achieve high dynamic steering control qualities requires a high installed power requirement of the HST, installation of the hydraulic power aggregate and radiator cooling system in the vehicle body. With significant constraints in terms of layout and volume-mass parameters, the installed power requirement of the hydrostatic transmission of the steering control system is limited. In connection with this, a number of engineering design solutions are being introduced in domestic and foreign control systems to create multi-stream drives that provide for dynamic properties increase and controllability of the high-speed tracked vehicle [1].

\footnotetext{
* Corresponding author: ig tar@mail.ru
} 
Severe conditions for vehicle operation, limited possibilities to organize service maintenance, insufficient reliability of electronic devices and hydrostatic transmissions, as well as limit on the cost of the steering control system lead to various proposals for creating simpler systems. These systems have advantages not only due to the abovementioned characteristics, but also in terms of weight and volume characteristics, simplicity, cost, degree of design and technological efficiency of structural elements [2]. Such control systems have discrete properties, many previously developed vehicles are equipped with them, including a modern promising transport vehicle TM-140, designed for severe conditions of operation in the Arctic region.

The results of comparative tests for tracked vehicles with various steering control systems (SCS) are shown in Fig. 1 [1,3]. The graphs show that with vehicle power-toweight ratio up to $20 \mathrm{~kW} / \mathrm{t}$, the vehicle average speed equipped with the SCS with discrete properties (graph 1 in Figure 1) is not lower than for vehicles with continuous ones (graph 3 in Figure 1). When driving along hard surface roads (asphalt, concrete, frozen ground), with limited road adhesion properties and intensive changes in curvature, on the test tracks such as «slalom», «elk test» and «long corner», speed characteristics of vehicles, equipped with SCS with discrete properties, are limited and do not exceed $36 \ldots 38 \mathrm{~km} / \mathrm{h}$ (graph 2 in Figure 1). It can be explained by impossibility of smooth control of trajectory curvature, angular acceleration, trajectory deviation compensation. At the same time, the intensity of driving activity increases, the number of SCS actuations (precision steering acts) per kilometer increases by 6 ... 9 times, reaching 96 . This value of control inputs is close to the maximum permissible number of acts per kilometer of distance (according to the driver's psychological capabilities). In this regard, compensation driving is effective at speeds less than $36-38 \mathrm{~km} / \mathrm{h}$, and with remote control of robotic systems installed in these vehicles, the average speed does not exceed $5 \mathrm{~km} / \mathrm{h}$. This identify the importance of speed increase in tracked vehicles equipped with SCS with discrete properties.

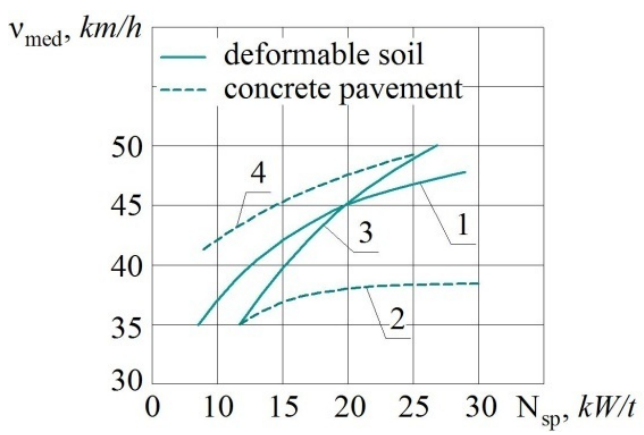

Fig. 1. Dependence of the average speed on the power-to-weight ratio of the vehicle with various steering control systems: 1, 2 - with discrete properties; 3, 4 with continuous properties

Thus, the goal is to provide the scientific substantiation of ways for increasing the degree of implementation of potential high-speed qualities of tracked vehicles with the discrete steering control system.

\section{Analysis of the principal factors limiting tracked vehicle high- speed performance and substantiation of the way of its improvement}

The rationale is based on the analysis of the research findings in controlled motion dynamics, which is performed in compliance with a mathematical model that has a physical meaning when stability conditions are fulfilled. At unstable motion, perturbations, 
including random ones, lead to significant and rapid deviations of the trajectory parameters that the driver detects with delay. Then, there is a delay in making a control input and only after that trajectory parameters are adjusted. Motion control in case of instability requires the driver's high qualification, leads to a sidewise skidding, swerving off the road, the driver's fatigue and, in all cases, to a speed decrease according to safety conditions. When driving at high speed, stability decreases due to centrifugal force during turning. The conditions of static stability are usually determined by an inequality of the derivatives of yaw moment $\mathrm{Mn}$ and cornering resistance moment $\mathrm{Mc}$ regarding/with respect to curvature $k=\frac{\dot{\varphi}}{V}: \quad \frac{\partial M_{C}}{\partial \mathrm{k}}>\frac{\partial M_{\Pi}}{\partial \mathrm{k}}$, where the cornering resistance moment is equal to $\mathrm{M}_{\mathrm{C}}=\frac{\dot{\varphi}}{v} \sum_{i=1}^{n} C_{y_{i}}\left(l_{i}-\chi\right)^{2}$, and longitudinal displacement of the center of turning $\chi=\frac{V^{2} L k}{2 \mu g}$ [4], $\dot{\varphi}$ is angular rotation rate, $v$ is the linear travel speed, $C_{y_{i}}$ is the coefficient of sidewise resistance of the lower rollers numbered $n, l_{i}$ is a longitudinal coordinate of the $i^{\text {th }}$ pair of lower rollers, $L$ is the length of the vehicle bearing surface, $\mu$ is the coefficient of resistance to rotation of the ground, $g$ is the gravitational acceleration.

The cornering resistance moment $\mathrm{Mc}$ is variable and depends on the curvature $k$, squared velocity $v$. For large values of the velocity $v$ with increasing trajectory curvature, the cornering resistance moment increases up to a certain value, and later, an increase in curvature is accompanied by a decrease in moment Mc (graph 1 in Fig. 2). Yaw moment $\mathrm{Mn}$, created by the engine and the steering mechanism with discrete properties, does not depend on trajectory curvature (graph 2 in Fig. 2). In the zone of increase in the moment of resistance, the derivative is positive, and in the zone of its decrease, it is negative. Taking into account that $\frac{\partial M_{\Pi}}{\partial \mathrm{k}}=0$ is the difference of the derivatives (graph 3 in Fig. 2) in the first zone is positive (the motion is stable), and in the second one is negative (the motion is unstable). Similarly, it is possible to determine the stability boundary for different speeds $v_{4}>v_{3}>v_{2}>v_{1}$, to plot the limit of speed stability $v$ as a function of trajectory curvature $k$ (graph 4 in Fig. 2). The limit of sidewise skidding speed $v<\sqrt{\frac{\mu g}{k}}$ is characterized by the graph 5 in Fig. 2. It is determined by a zero value of the cornering resistance moment or the longitudinal displacement of the center of turn, reaching the value $\chi=0,5 \mathrm{~L}$. The given findings of experimental research of the dynamics of controlled motion along a deformable soil [4] show that the longitudinal displacement of the center of turn by a value $\chi$ leads to sidewise motion. In this case, a slip angle $\theta$ (a directional angle) is formed between the longitudinal axis of the vehicle and the line tangent to a desired trajectory. When the value $\chi$ reaches a value of $0.5 \mathrm{~L}$, the motion remains controllable, and the yaw moment $\mathrm{Mn}$, despite a generally accepted opinion, does not equal to zero. This is caused by a sidewise motion that increases the resistance moment by a value $\theta$. $\sum_{i=1}^{n} C y_{i}\left(l_{i}-\chi\right)$ (the shaded area in graph 1, figure 2). This circumstance makes it possible to expand the zones of controlled and stable motion (the shaded areas in Graphs 4 and 5, Fig. 2).

When driving along hard ground with low adhesion properties, the increase in the component of the moment Mc, formed by sidewise motion, is insignificant. Under these conditions, stability can be ensured by appropriate control of the power-train unit (turbine revolutions by unblocking the torque converter and transiting the engine to the regulatory engine mechanism). This ensures the creation of the traction function $P_{2}$ on the leading track under stability condition. When turning with the steering brake control mechanism in the form of «tear» mechanism [5] controlled by an electric motor, it is possible to control the force $P_{1}$ from the condition of ensuring the required dependence $\frac{\partial M_{\Pi}}{\partial \mathrm{k}}=0,5 \mathrm{~B} \frac{\partial\left(P_{2}-P_{1}\right)}{\partial \mathrm{k}}<$ $\frac{\partial M_{C}}{\partial \mathrm{k}}$ (line 6 in Fig. 2). 
Another factor limiting high-speed performance, as shown by the analysis of the experimental findings obtained in studying the controlled motion dynamics [1] and experience of driving vehicles along the roads with an intensive change in trajectory curvature, is implementation of potential speed performance, which is largely limited by the dynamic properties of the vehicle, i.e. by the phase lag of the response to the control input (Fig. 3). It is manifested in the fact that the longitudinal axis of the vehicle body does not have time to turn relatively to the tangent of the desired trajectory. To fit into a required corridor, it is necessary to reduce travel speed.

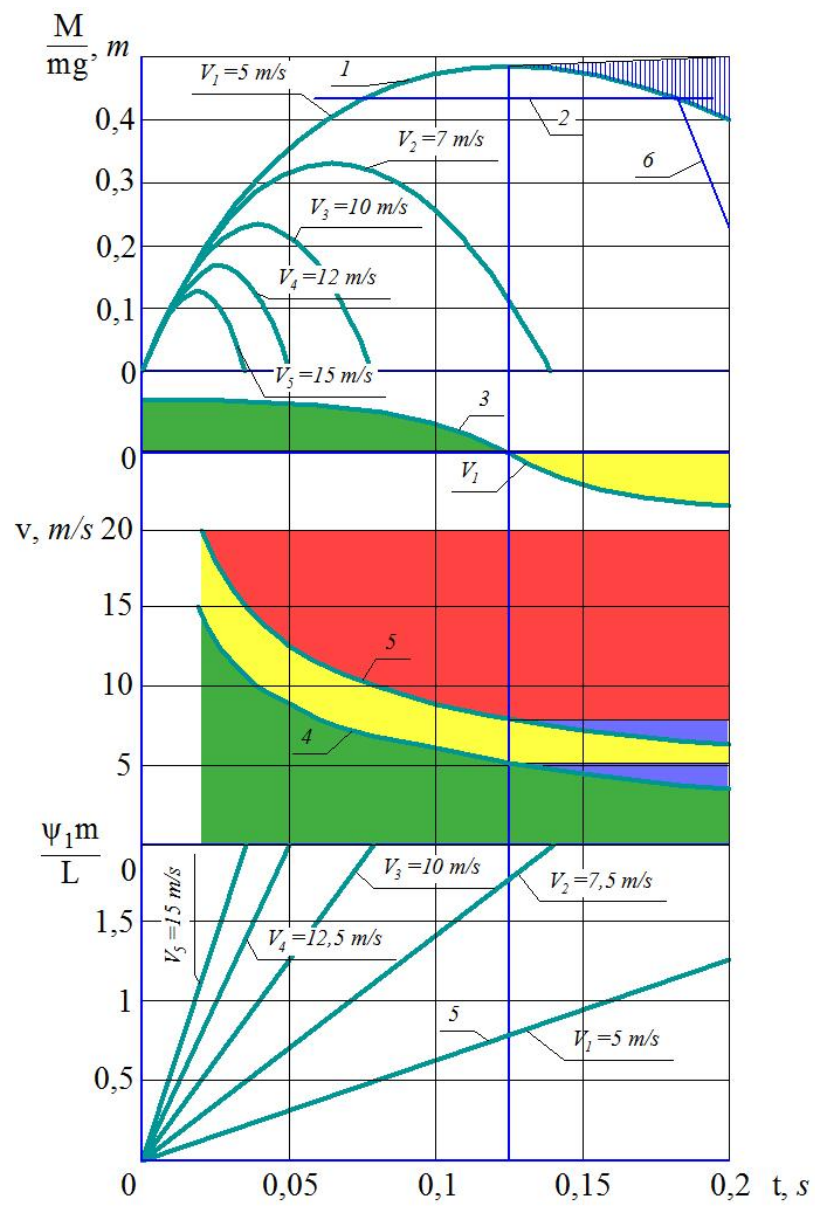

Fig. 2. Evaluation of static stability of tracked vehicle motion when turning. 


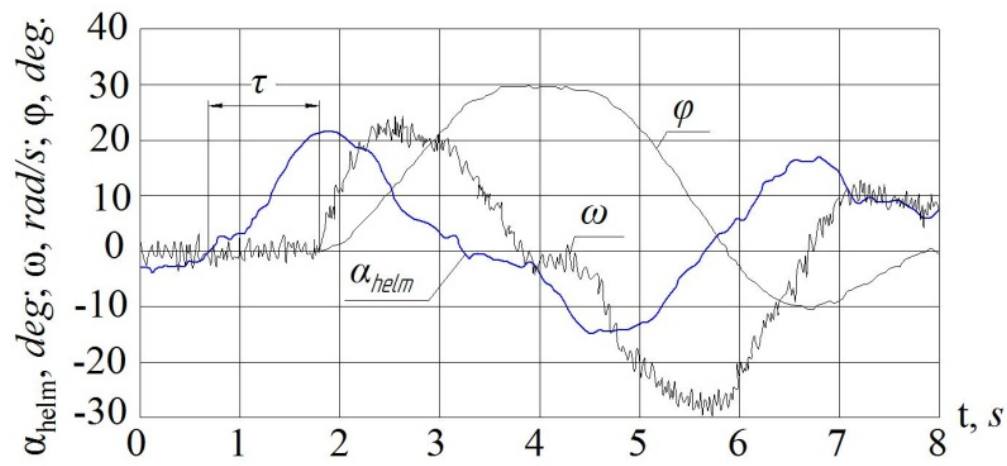

Fig. 3. Fragment of the oscillogram with a record of the change in kinematic parameters during driving «slalom» (deformed ground, half-wave length $\Delta \mathrm{S}=30 \mathrm{~m}$, IV gear): $\alpha$ is steering angle; $\omega$ is angular velocity; $\varphi$ is a heading angle (an azimuth)

The rationale for the possibility of increasing the speed performance of a tracked vehicle by reducing the phase lag of response to a harmonic control input is shown in [1].

It should be noted that the results of the experimental determination of transient characteristics differ by greater oscillation index (large values of oscillation amplitudes relative to the steady-state value), which is due to neglecting the elasticity of the elements in the mathematical model. To increase the convergence of the results, the calculated stiffness is introduced into the differential equation of rotational motion. The magnitude of this stiffness can be considered as a composition of the following components: deformation of the connecting shafts; operating track runs; elements of a lower roller suspension unit, and also stretching of the lagging slack upper track runs on the upper rollers. At turning, the tracked vehicle, the value of the calculated stiffness depends on the transmission ratio (the number of the engaged gear), this calculated stiffness is essentially non-linear and asymmetrical, which can excite parametric oscillations. This paper regards the reduced stiffness as linearized. Taking into consideration the abovementioned, the convergence of the results of experimental research and modeling rises.

For tracked vehicles with a stepped steering mechanism that provides for discrete control of driving direction, the angular velocity $\omega$ undergoes a second-kind discontinuity, and its derivative $\dot{\omega}$ is a delta function $\delta(t)$, i.e. in absolute value, it can vary in an unlimited range. However, from the experimental data, it follows that in the elastically inertial mechanical system (track assembly - transmission), including frictional elements and interacting with the soil in a way of slipping, the values of the derivative $\dot{\omega}$ varies continuously in finite, albeit significant, limits. Even with a limited deviation of angular velocity up to $5 \mathrm{deg} / \mathrm{s}$ with a frequency of $2.5 \mathrm{~Hz}$ (due to skidding and oscillatory processes in the power train unit), the angular accelerations reach $32 \mathrm{deg} / \mathrm{s}^{2}$, and at on-off cycling of the mechanism in the long turns - up to $74 \mathrm{deg} / \mathrm{s}^{2}$. These values significantly exceed the angular accelerations when the tracked vehicle turns with a differential hydrostatic steering mechanism, the yaw moment of which is created by the hydraulic motor. Therefore, when driving along deformable soils, the speed characteristics of vehicles with discrete properties of the steering control system may be higher than (with HSSM hydroststatic steering mechnaism). In this case, implementation of high values of sideways accelerations (up to $10 \ldots 11 \mathrm{~m} / \mathrm{s}^{2}$ ) is not accompanied by skidding, because after several test runs, the rutted track with depth up to $60 . .80 \mathrm{~mm}$ is formed. However, when moving on poorly deformable soils, these possibilities cannot be implemented because of the limited adhesion properties.

The abovementioned findings show that the discrete properties of the steering control system limit the vehicle speed due to oscillativity of transients and significant overshoot when going into corner. In technology, these phenomena are usually called the Bonanza 
effect $[7,8,9,10]$, which is observed in the process of mechanical systems control of transport vehicles. In general, the resulting oscillations limit vehicle speed, they are perceived by the driver as alarming, increase the load on the structural elements and worsen the level of comfort. Amplitudes of these oscillations must be reduced by frequency detuning, i.e. the maximum amplitudes will be shifted to frequencies that are not critical for the given vehicle, or by the method of redistribution of control inputs, i.e. the control pulse, which causes oscillations when applying torque, can be changed to prevent or reduce the formation of free oscillations. These methods of oscillation damping are described in detail in $[7,13]$.

The analysis of applying various regulators showed that the best results are demonstrated by ZV-shapers, which allow for minimizing the limiting overshoot up to 5\%, and the transient becomes close to aperiodic, including when traveling at high speed on soils with low adhesion properties.

It should be noted that the research is based on the analysis of the linear system. An urgent task for future research is to refine models of the controlled motion dynamics of tracked vehicles, when the elastic characteristics of the tracked propulsion assembly are nonlinear and under the condition of excitation of wave processes in the elastic caterpillar track.

\section{Conclusions}

As a result of modeling and experimental study of the dynamics of the controlled motion of the tracked vehicle with discrete properties of the steering control system, it is established that the degree of realization of potential speed qualities is limited by the following dynamic properties of the vehicle:

- static stability of the vehicle motion at turning and sidewise skidding;

- phase lag of vehicle response to a harmonic control input;

- the quality of response to a steering jerk (per individual control input).

To increase vehicle speed, the ways to improve motion stability are substantiated by satisfying the condition that the difference of the partial derivatives is positive, with respect to curvature of trajectory of yaw and resistance moments. This can be achieved by running the engine at the regulatory branch, by unlocking the torque converter and by controlled skidding of steering brake mechanism of the discs. The zone of motion stability can be expanded by sidewise motion of the vehicle due to an additional component of the cornering resisting moment.

The operation of a vehicle high speed limit on roads with an intensive change in curvature, which is determined by phase delay in vehicle response to a control action, is proposed to be reduced by increasing the linear stiffness of the rubber-metal hinges of the caterpillar track and by introducing a differentiating device into the control system.

On the roads with limited adhesion properties, the speed characteristics can be increased by introducing a ZV Shaper filter in the steering brake control unit, which prevents from excitation of oscillations, while the overshoot does not exceed $5 \%$.

This study was funded by the program of the President of the Russian Federation for the support of young scientists - Grant MK-5809.2018.8, and also with partial support under the state task IES UB RAS No. 0391 - 2014 - 007. 


\section{References}

1. Derzhansky V. B., Taratorkin I.A., Forecasting of Dynamic Loading of Hydromechanical Transmissions of Transport Vehicles: Monograph - Yekaterinburg: Ural RAN, - 176, (2010)

2. Proekt koncepcii ustojchivogo razvitiya arkticheskoj zony [The draft concept of sustainable development of the Arctic zone of the Russian Federation] - Moscow, Minregion publ., (2005)

3. J.Y. Wong, International Journal of Vehicle Mechanics and Mobility., 28 (1997), 197219, 10.1080/00423119708969354

4. A. Taratorkin, V. Derzhanskii, I. Taratorkin, Procedia Engineering, 136, 1368-1377, (2016), DOI 10.1016/j.proeng.2016.07.331

5. A. Gurevich, V. Kornev, A. Khromov, Theory of automatic control in examples and problems with solutions in MATLAB, 464, (2016)

6. R. Fischer, F. Küçükay, G. Jürgens, R. Najork, B. Pollak, DOI 10.1007/978-3-31905263-2, (2015)

7. G. Jurgens, R. Fischer, 697, VDI-Berichte, Dusseldorf, 233, (1988)

8. H-J Schspf, G Jurgens, R Fischer, 91, ATZ, 568-575, (1989)

9. K.L. Sorensen, A Thesis Presented to The Academic Faculty, ( 2008)

10. K.L. Sorensen, W.E, Singhose, S. Dickerson, 15, № 7, 825-837, (2007)

11. A.P. Kuznetsov, A.V. Markov, A.S. Shmarlouski, T.V. Gavrilik, Doklady BGUIR, 6(60), 5-12, (2011)

12. I. Taratorkin, V. Derzhanskii, A. Taratorkin, MATEC Web of Conferences 129, 06029 (2017) DOI: 10.1051/matecconf/201712906029 